\title{
Isothermal Martensitic Transformations in an Aged Ni-Rich Ti-Ni Alloy Containing Coherent $\mathrm{Ti}_{3} \mathrm{Ni}_{4}$ Particles
}

\author{
Hiroshi Akamine ${ }^{1}$, Yohei Soejima ${ }^{2, * 1}$, Tadaaki Nakamura ${ }^{2, * 1}$, Farjami Sahar ${ }^{3}$, Takashi Fukuda ${ }^{4}$, \\ Tomoyuki Kakeshita ${ }^{5}$ and Minoru Nishida ${ }^{1, * 2}$ \\ ${ }^{1}$ Department of Advanced Materials Science and Engineering, Faculty of Engineering Sciences, Kyushu University, \\ Kasuga 816-8580, Japan \\ ${ }^{2}$ Department of Applied Science for Electronics and Materials, Interdisciplinary Graduate School of Science and Engineering, \\ Kyushu University, Kasuga 816-8580, Japan \\ ${ }^{3}$ Department of Materials Science and Engineering, University of Michigan, Ann Arbor, MI 48109, USA \\ ${ }^{4}$ Department of Materials Science and Engineering, Graduate School of Engineering, Osaka University, Suita 565-0871, Japan \\ ${ }^{5}$ Department of Mechanical Engineering, Faculty of Engineering, Fukui University of Technology, Fukui 910-8505, Japan
}

The time dependence nature of B2 (ordered cubic) to R (trigonal) and R to B19' (monoclinic) martensitic transformations in an aged Nirich Ti-Ni alloy was investigated by electrical resistivity measurements. Both B2 to R and R to B19' transformations exhibit time dependence nature in the aged alloy containing coherent lenticular $\mathrm{Ti}_{3} \mathrm{Ni}_{4}$ precipitates with an average diameter of about $110 \mathrm{~nm}$. However, the successive $\mathrm{B} 2-\mathrm{R}-\mathrm{B} 19^{\prime}$ isothermal transformation is not detected by electrical resistivity measurements. The isothermal R-B19' transformation is visualized by in situ scanning electron microscopy (SEM) observations. [doi:10.2320/matertrans.MT-MJ2019004]

(Received June 25, 2019; Accepted August 19, 2019; Published September 30, 2019)

Keywords: shape memory alloys, titanium-nickel alloy, scanning electron microscopy, martensitic phase transformation, precipitation

\section{Introduction}

The isothermal B2-B19', B2-R, and R-B19' martensitic transformations in $\mathrm{Ti}-\mathrm{Ni}$ based alloys have been investigated in several studies. ${ }^{1-6)}$ The kinetics of these three transformations was examined by Kustov et al. for Ti-50.2Ni and Ti-48Ni-2Fe (at\%) alloys with various heat treatments. ${ }^{2)}$ They reported that the B2-B19' and R-B19' transformations have isothermal nature, whereas the $\mathrm{B} 2-\mathrm{R}$ transformation does not. Fukuda et al. found that the B2-B19' transformation in Ti-51.2 and $51.3 \mathrm{Ni}($ at $\%)$ alloys and the B2-R transformation in $\mathrm{Ti}-44 \mathrm{Ni}-6 \mathrm{Fe}$ (at\%) alloy are isothermal. ${ }^{3,4,6)}$ These three alloys consisted of a B2 single phase quenched from $1273 \mathrm{~K}$. Ji et al. reported that the isothermal B2-R transformation takes place in the Ti-51.3Ni (at\%) alloy with holding above the martensite start temperature $\left(M_{s}\right)$ of the B2-B19' transformation. ${ }^{5}$ )

Shape memory and superelastic properties have been improved by the precipitation strengthening of $\mathrm{Ti}_{3} \mathrm{Ni}_{4}$ particles. ${ }^{7)}$ The successive B2-R-B19' transformation occurs in Ni-rich $\mathrm{Ti}-\mathrm{Ni}$ alloys containing $\mathrm{Ti}_{3} \mathrm{Ni}_{4}$ particles. ${ }^{7)}$ However, there are no reports of whether the B2-R and RB19' transformations in aged Ni-rich $\mathrm{Ti}-\mathrm{Ni}$ alloys have isothermal nature. In the present study, we investigate the time dependence nature of B2-R and R-B19' transformations in an aged Ni-rich $\mathrm{Ti}-\mathrm{Ni}$ alloy containing coherent $\mathrm{Ti}_{3} \mathrm{Ni}_{4}$ particles. In situ scanning electron microscopy (SEM) observations are also performed to visualize the progress of the isothermal R-B19' transformation.

\section{Experimental Procedure}

An ingot of the $\mathrm{Ti}-51.0 \mathrm{Ni}($ at $\%)$ alloy of nominal

\footnotetext{
${ }^{* 1}$ Graduate Student, Kyushu University

${ }^{* 2}$ Corresponding author, E-mail: nishida.minoru.355@m.kyushu-u.ac.jp
}

composition was prepared by an arc melting method and remelted several times to ensure homogeneity. The ingot was heat-treated at $1273 \mathrm{~K}$ for $38.4 \mathrm{ks}$ in a vacuum and quenched into ice water. Rectangular plates $(10 \times 2 \times$ $0.2 \mathrm{~mm}$ ) for electrical resistivity measurements and square plates $(3 \times 3 \times 1 \mathrm{~mm})$ for differential scanning calorimetry (DSC) and SEM were cut from the ingot and solution-treated at $1173 \mathrm{~K}$ for $3.6 \mathrm{ks}$ in a vacuum with pure $\mathrm{Zr}$ as a getter metal, followed by quenching into ice water. The plates were aged $773 \mathrm{~K}$ for $3.6 \mathrm{ks}$ in the same atmosphere used for the solution treatment to avoid the multistage martensitic transformation (MMT) ${ }^{8-10)}$ and quenched into ice water. The surface of each specimen was electropolished in an electrolyte of methanol and sulfuric acid (4:1 v/v) around $273 \mathrm{~K}$.

The electrical resistivity was measured by the four-probe method with a current of $100 \mathrm{~mA}$ and a frequency of $17 \mathrm{~Hz}$. The specimen for the resistivity measurement was mounted on a $\mathrm{Cu}$ stage using Apiezon $\mathrm{N}$ grease, and the temperature of the stage was monitored throughout the measurements. The overshoot and undershoot of the temperature before isothermal holding was within $200 \mathrm{mK}$, and the fluctuation of the temperature during holding was within $10 \mathrm{mK}$. The DSC measurements were made in the temperature range between 353 and $123 \mathrm{~K}$ with a cooling and heating rate of $5 \mathrm{~K} \mathrm{~min}^{-1}$. The characteristic temperatures of the transformation events were determined by the extrapolation method from the electrical resistivity and DSC curves. A field-emission SEM (ULTRA 55, ZEISS) equipped with an annular backscattered electron (BSE) detector and a Peltier stage (Coolstage, Deben) with a working temperature of 220-320 K were used for the in situ SEM observations. ${ }^{9-11)}$ The temperature stability of the Peltier stage was about $200 \mathrm{mK}$ during isothermal holding. 


\section{Results and Discussion}

There were two exothermic peaks derived from the B2-R and R-B19' transformations in the DSC curve of the aged alloy upon cooling. The starting temperatures of B2-R and R-B19' exothermic reactions were 289 and $238 \mathrm{~K}$, respectively. These temperatures agree well with those estimated from the electrical resistivity measurements as described later. In contrast, a single endothermic peak was observed upon heating because of the difference in thermal hysteresis between the B19'-R and R-B2 transformations. ${ }^{7-10)}$ The results confirm that there was no $\mathrm{MMT}^{8-10)}$ in the present aged specimen.

Figure 1 shows the electrical resistivity curves measured with a cooling and heating rate of $2 \mathrm{~K} \mathrm{~min}^{-1}$. During cooling, the resistivity started to gradually increase at $307 \mathrm{~K}$, and then

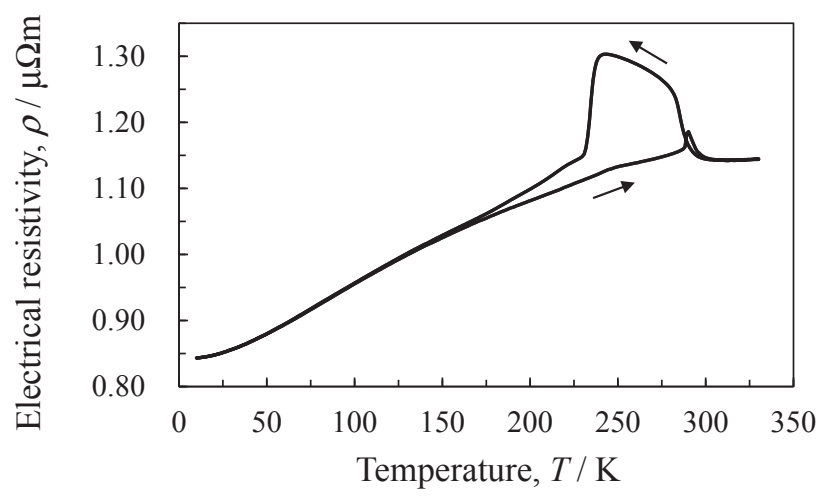

Fig. 1 Temperature dependence of the electrical resistivity of a Ti-51.0Ni alloy aged at $773 \mathrm{~K}$ for $3.6 \mathrm{ks}$ after solution treatment at $1173 \mathrm{~K}$ for $3.6 \mathrm{ks}$. drastically increases at $290 \mathrm{~K}$. The first increase corresponds to the starting temperatures of the B2-R exothermic reaction in the DSC cooling curve. There is a large decrease in resistivity from about $238 \mathrm{~K}$ due to the R-B19' transformation. These results are consistent with the DSC results. Figure 2 shows SEM-BSE images of the aged specimen. The average grain size of the parent B2 phase is about $20 \mu \mathrm{m}$ in diameter (Fig. 2(a)). The lenticular $\mathrm{Ti}_{3} \mathrm{Ni}_{4}$ precipitates of about $110 \mathrm{~nm}$ in average diameter are homogeneously distributed around the grain boundary (Fig. 2(b)) and grain interior (Fig. 2(c)). There is no largescale microstructural heterogeneity in the precipitation morphology, distribution and size, which is responsible for the MMT. ${ }^{8-10,12-14)}$

To detect the isothermal B2-R transformation, the specimen was held at set temperatures for $3.6 \mathrm{ks}$ after cooling from $330 \mathrm{~K}$ at a rate of $2 \mathrm{~K} \mathrm{~min}^{-1}$ in each holding cycle (Fig. 3(a)). There is an increase in resistivity during holding at $287 \mathrm{~K}$ (blue dots and line), and a transformation hysteresis of approximately $3 \mathrm{~K}$ between cooling and heating curves. The increase in resistivity and narrow hysteresis within $5 \mathrm{~K}$ are characteristic features of the B2-R transformation. These results suggest that the isothermal B2-R transformation proceeds during holding at $287 \mathrm{~K}$. Figure $3(\mathrm{~b})$ shows the results of isothermal holding experiments at set temperatures for $3.6 \mathrm{ks} . \Delta \rho$ is the difference in the electrical resistivity values between the start of the measurement and an arbitrary time at the set temperatures. The other set temperatures were 294, 280, 270, 265, 260, 255, and $250 \mathrm{~K}$. The increase in resistivity is clearly observed between 294 and $270 \mathrm{~K}$. Therefore, the isothermal B2-R transformation proceeds in this temperature range. However, there is no
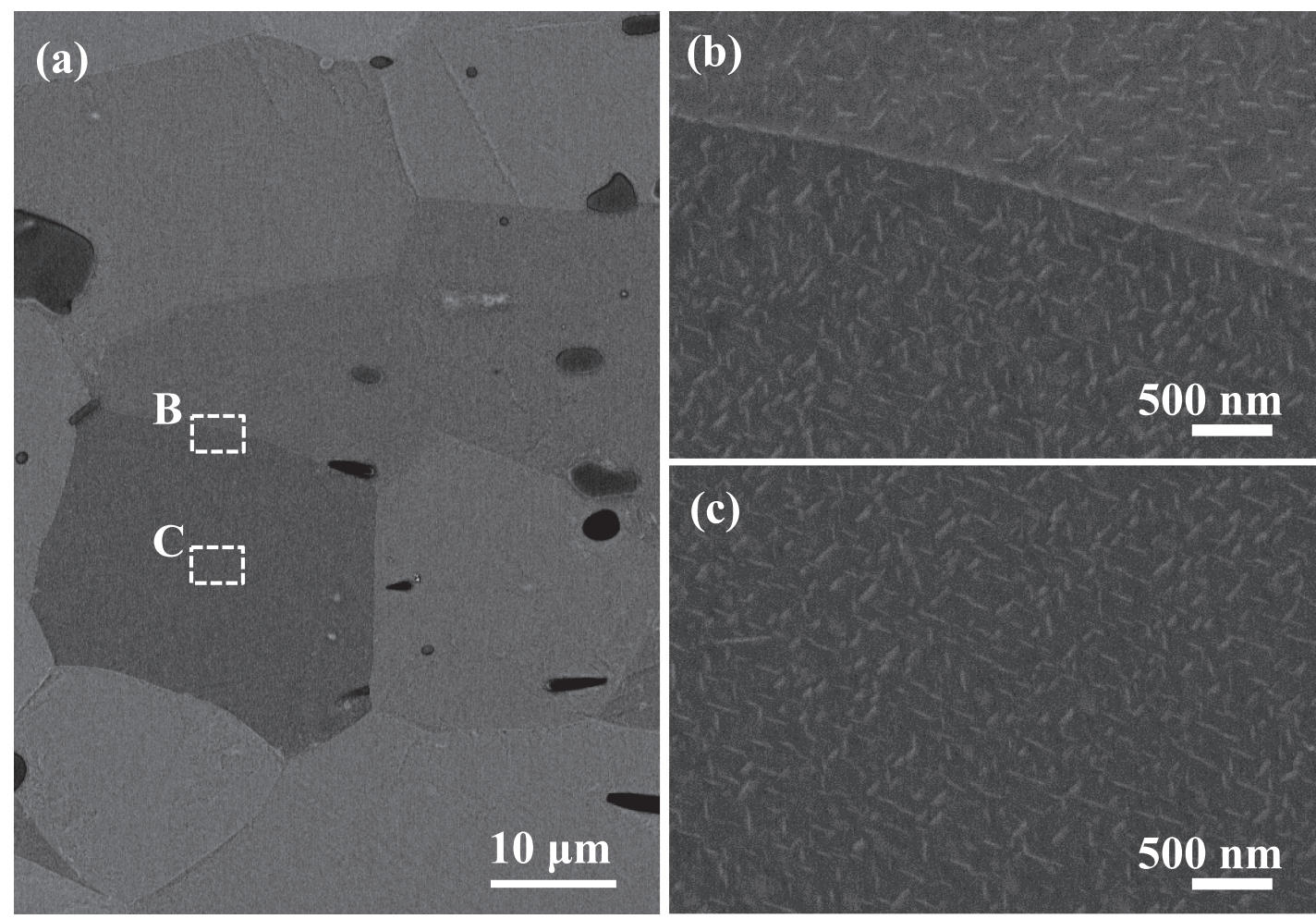

Fig. 2 (a) Low-magnification SEM-BSE image. (b), (c) Enlarged SEM-BSE images taken from areas B and C in (b), showing the homogenous distribution of $\mathrm{Ti}_{3} \mathrm{Ni}_{4}$ precipitates around the grain boundary and interior. 

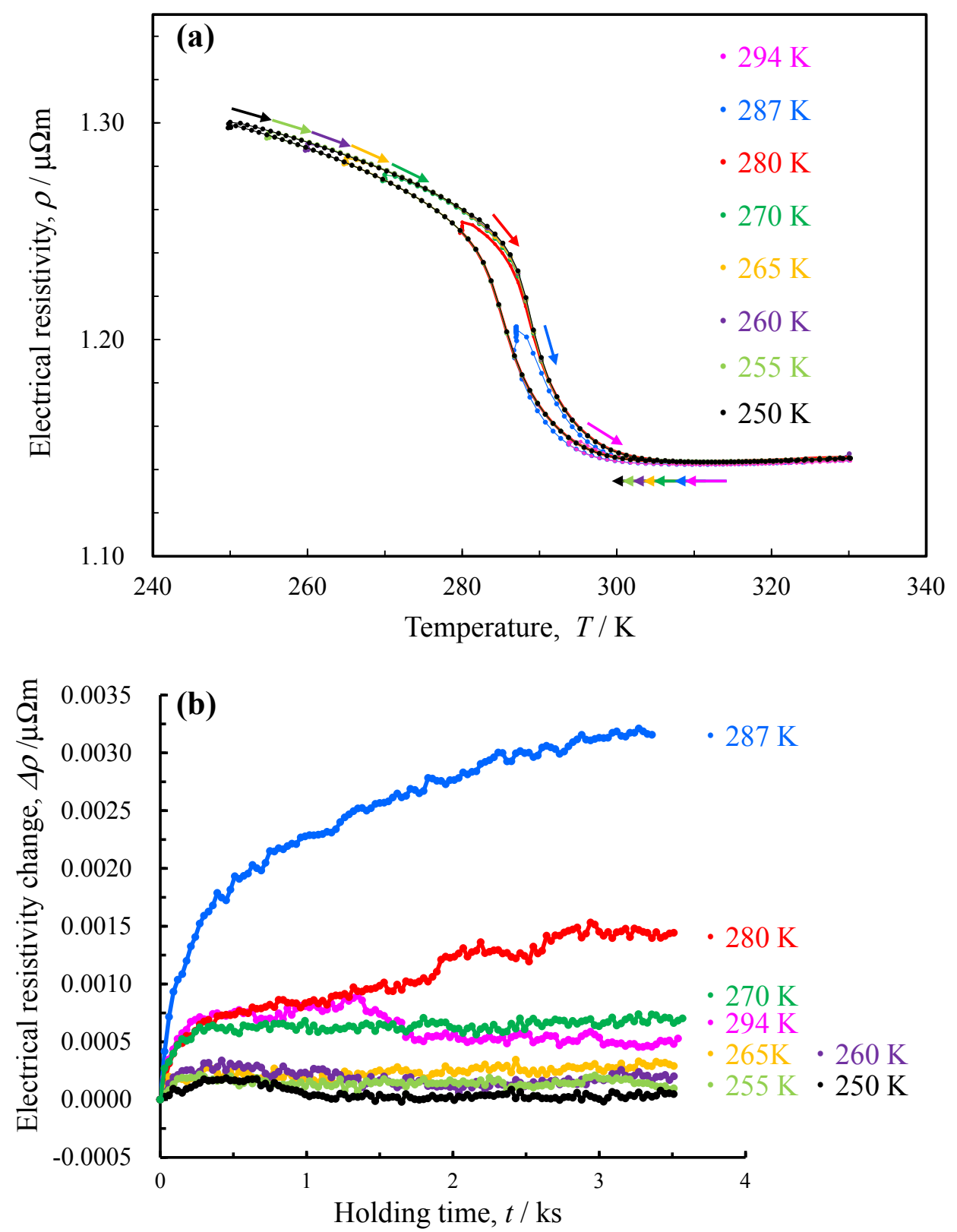

Fig. 3 (a) Temperature dependence of the electrical resistivity of the aged specimen. Results of isothermal holding experiments at 294 , $287,280,270,265,260,255$, and $250 \mathrm{~K}$. The specimen was heated to $330 \mathrm{~K}$, cooled to each temperature at a rate of $2 \mathrm{~K} \mathrm{~min}^{-1}$, and then held for $3.6 \mathrm{ks}$. (b) Time dependence of electrical resistivity during holding at the temperatures indicated in (a).

change in resistivity between 265 and $250 \mathrm{~K}$. The successive B2-R-B19' isothermal transformation is not detected above $250 \mathrm{~K}$.

Figure 4(a) shows the results of isothermal holding experiments at set temperatures for $3.6 \mathrm{ks}$. The heating, cooling, and holding procedures were the same as those of the B2-R transformation. There was no thermal cycle effect, namely, no decrease in transformation temperatures of the B19' martensite as discussed later, because the $\mathrm{Ti}_{3} \mathrm{Ni}_{4}$ particles suppress the dislocation formation during the transformation cycles. ${ }^{1,15,16)}$ The set temperatures were 246 , $244,242,240,238$, and $228 \mathrm{~K}$. The vertical decrease in each of the resistivity curves at the set temperatures is due to the progress of the R-B $19^{\prime}$ transformation during the holding. Figure 4(b) shows the time dependence of $\Delta \rho$ of the specimen at the set temperatures. The size and rate of the resistivity change increase with as the set temperature is lowered above the R-B19' transformation start temperature of $238 \mathrm{~K}$, whereas they decreased at $228 \mathrm{~K}$. The resistivity started to decrease as soon as the holding started at all the set temperatures. This suggests that the transformation occurs without detectable incubation time in the aged specimen, although Fukuda et al. reported an incubation time in the isothermal B2-B19' transformation in quenched Ti-51.3 (at\%) alloy. ${ }^{6}$ ) After the isothermal holding at $228 \mathrm{~K}$ and subsequent heating to $330 \mathrm{~K}$ in Fig. 4(a), the cyclic isothermal holding experiments at $238 \mathrm{~K}$ were completed 10 times. Figures 5(a) and (b) shows the result of first and tenth cycles, respectively. The size and rate of the resistivity change in Figs. 5(a) and (b) are fairly identical to those at $238 \mathrm{~K}$ in Fig. 4(a). It is confirmed that there is no thermal cycle effect upon the cyclic isothermal holding experiments. 


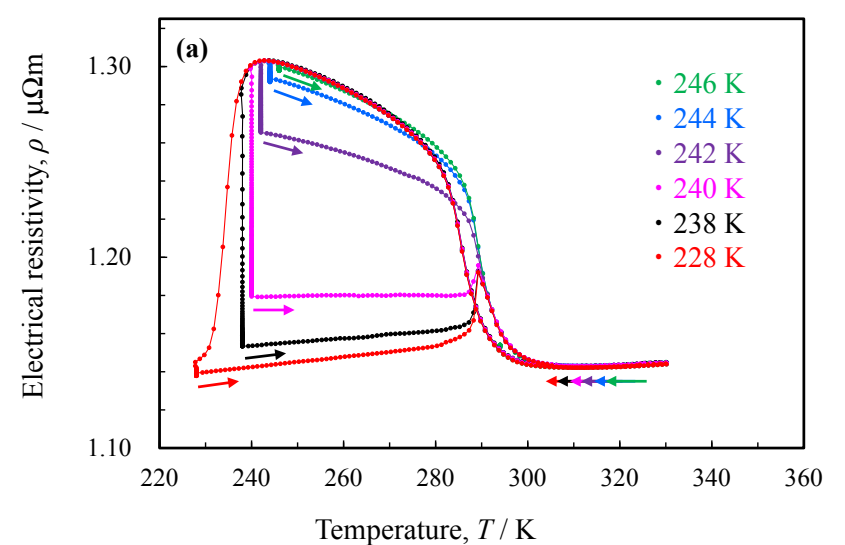

(b)

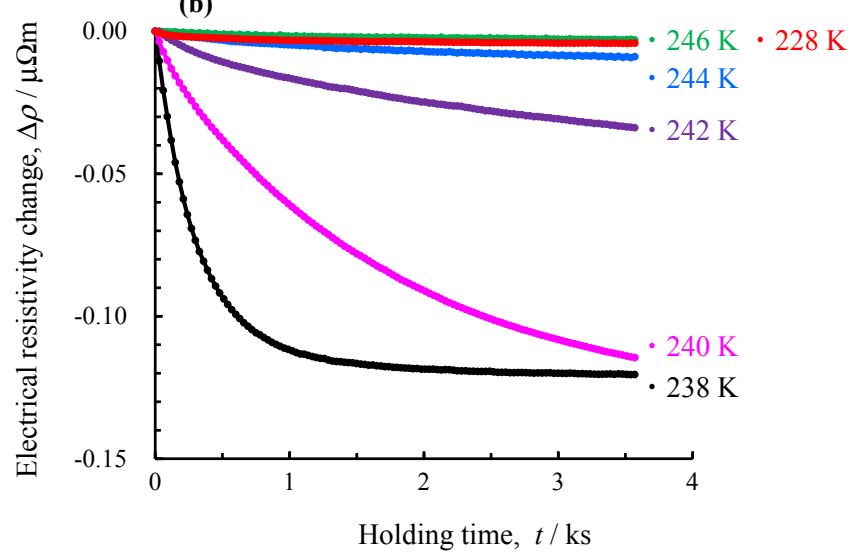

Fig. 4 (a) Temperature dependence of the electrical resistivity of the aged specimen. Results of isothermal holding experiments at 246, 244, 242 , 240,238 , and $228 \mathrm{~K}$ for $3.6 \mathrm{ks}$. (b) Time dependence of the electrical resistivity during holding at the temperatures indicated in (a).

Thus, the present results are sufficiently reliable and reproducible for investigating the isothermal R-B19' transformation.

It is hard to discuss directly and quantitatively the differences and similarities of the time dependence nature of transformations in solution treated and aged alloys, since it is difficult to prepare and adjust the solution treated and aged alloys with the same B2-R and R-B19' transformation temperatures. Comparing Fig. 3 in the present study with Figs. 2 and 3 in the Ref. 4), the resistivity increment size and rate in the present aged alloy held at $287 \mathrm{~K}$ is larger than that in the solution treated $\mathrm{Ti}-44 \mathrm{Ni}-6 \mathrm{Fe}$ held at $150 \mathrm{~K}$. ${ }^{4)}$ These temperatures were located at the middle point of the starting and finishing temperatures of abrupt resistivity change with negative temperature coefficient. Comparing Fig. 4 in the present study with Figs. 1 and 2 in the Ref. 3), the resistivity decrement size and ratio with the R-B19' transformation in the aged alloy held at $238 \mathrm{~K}$ for $3.0 \mathrm{ks}$ is quite larger than those with B2-B19' transformation in the solution treated $\mathrm{Ti}-$ $51.2 \mathrm{Ni}$ held at $155 \mathrm{~K}$ for $3 \mathrm{ks} .{ }^{3)}$ The former temperature was the same as the starting temperatures of R-B19' exothermic reactions in the aged alloy as mentioned above. The latter one was $1 \mathrm{~K}$ lower than the starting temperatures of B2-B19' exothermic reactions in the solution treated alloy. ${ }^{3)}$ These facts suggest that both the isothermal transformations are easy to progress in the aged alloy with coherent precipitates.
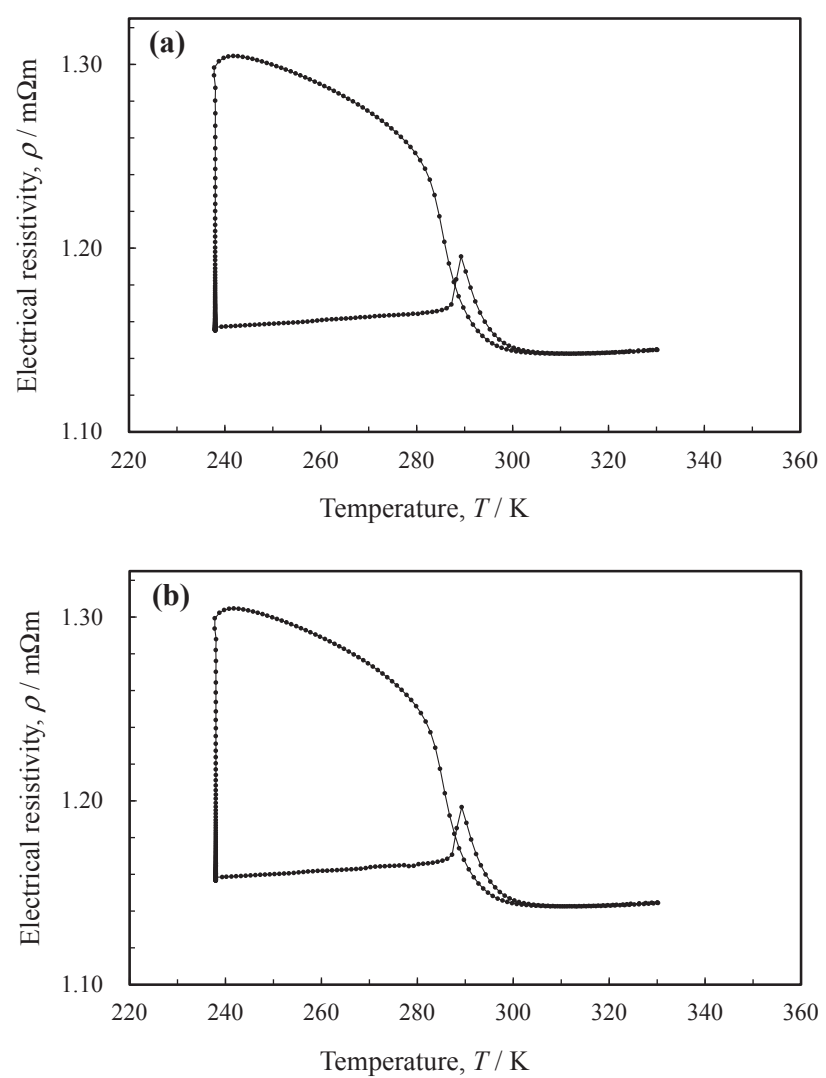

Fig. 5 Results of cyclic isothermal holding experiments at 238 K. (a) First cycle. (b) Tenth cycle. See text in details.

In other words, the coherent strain and/or stress between precipitates and matrix play an important role in the acceleration of isothermal transformations.

Figures 6(a)-(d) show a series of in situ SEM-BSE images during isothermal holding at $240 \mathrm{~K}$, which is $2 \mathrm{~K}$ higher than the R-B19' transformation start temperature shown in Fig. 1. The distribution and size of the $\mathrm{Ti}_{3} \mathrm{Ni}_{4}$ precipitates were homogeneous between the grain boundary and the grain interior (Figs. 2(a)-(c)), although the precipitates were not visible in the low-magnification images. There are nonmetallic inclusions with dark contrast at the grain boundary on the left-hand side. The energy-dispersive X-ray analysis identifies the inclusion as $\operatorname{TiC}(\mathrm{O}) .{ }^{17}$ The R-phase transformation was also not visible because its transformation strain is small compared with those of the B2-B19' and RB19' martensitic transformations. After holding for $1.8 \mathrm{ks}$, the $\mathrm{B} 19^{\prime}$ phase appears as bright contrast $\mathrm{t}^{9,10)}$ at the grain interior and boundary (arrows, Fig. 6(b)). Upon continuous holding for up to $10.8 \mathrm{ks}$, the amount of martensitic phase increases with increasing holding time (Figs. 6(c) and (d)). These observations clearly show that the R-B19' transformation exhibits time dependence nature in an aged Ni-rich $\mathrm{Ti}-\mathrm{Ni}$ alloy containing coherent particles of $\mathrm{Ti}_{3} \mathrm{Ni}_{4}$ from the metallographic point of view. So far as we know, the present results are the first visualization of isothermal nature of R-B19' transformation in Ti-Ni alloys, although there are coherent precipitates in the matrix.

In summary, the present results confirm that both B2-R and R-B19' martensitic transformations in an aged Ni-rich Ti-Ni alloy have isothermal nature, although the effect of coherency 

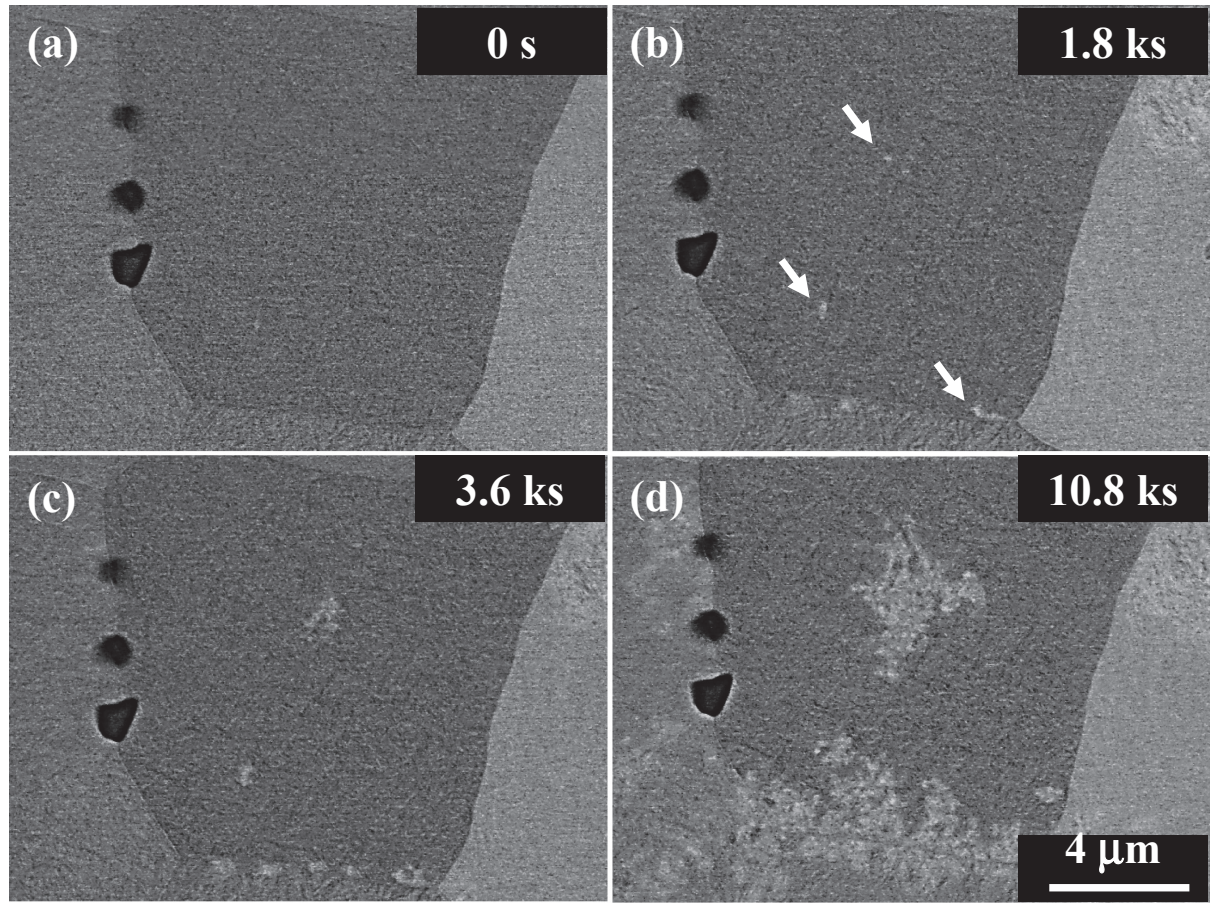

Fig. 6 Series of in situ SEM-BSE images during isothermal holding at $240 \mathrm{~K}$ for (a) 0, (b) 1.8, (c) 3.6, and (d) $10.8 \mathrm{ks}$. Arrows indicate the B19' phase.

between precipitates and matrix on the isothermal behaviors should be further investigated in details.

\section{Conclusions}

The time dependence nature of $\mathrm{B} 2$ to $\mathrm{R}$ and $\mathrm{R}$ to $\mathrm{B} 19^{\prime}$ martensitic transformations in an aged Ni-rich Ti-Ni alloy containing coherent lenticular $\mathrm{Ti}_{3} \mathrm{Ni}_{4}$ precipitates with an average diameter of about $110 \mathrm{~nm}$ was investigated by electrical resistivity measurements and in situ SEM observations. The results are summarized as follows:

(1) The starting temperatures of B2-R and R-B19' transformations were 289 and $238 \mathrm{~K}$, respectively.

(2) The isothermal B2-R transformation proceeds between 294 and $270 \mathrm{~K}$. The successive B2-R-B19' isothermal transformation is not detected by electrical resistivity measurements between 265 and $250 \mathrm{~K}$.

(3) The isothermal R-B19' transformation occurs below $246 \mathrm{~K}$ without detectable incubation time. The time dependence nature of R-B19' transformation is clearly visualized by in situ SEM observations. The isothermal B19' phase nucleates at both grain interior and boundary.

\section{Acknowledgement}

This work was partly supported by Grants-in-Aid for Scientific Research (A: No. 25249086 and No. 19H00829) from the Japan Society for the Promotion of Science and the Japan Science and Technology Agency (JST) (grant number:
20100113) under Industry-Academia Collaborative R\&D Program "Heterogeneous Structure Control: Towards Innovative Development of Metallic Structural Materials".

\section{REFERENCES}

1) K. Otsuka, X. Ren and T. Takeda: Scr. Mater. 45 (2001) 145-152.

2) S. Kustov, D. Salas, E. Cesari, R. Santamarta and J.V. Humbeeck: Acta Metall. 60 (2012) 2578-2592.

3) T. Fukuda, S. Yoshida and T. Kakeshita: Scr. Mater. 68 (2013) 984987.

4) T. Fukuda, M. Todai and T. Kakeshita: Scr. Mater. 69 (2013) 239-241.

5) Y. Ji, D. Wang, X. Ding, K. Otsuka and X. Ren: Phys. Rev. Lett. 114 (2015) 055701.

6) T. Fukuda, T. Kawamura and T. Kakeshita: J. Alloy. Compd. 683 (2016) 481-484.

7) K. Otsuka and X. Ren: Prog. Mater. Sci. 50 (2005) 511-678.

8) M. Nishida, T. Hara, T. Ohba, K. Yamaguchi, K. Tanaka and K. Yamauchi: Mater. Trans. 44 (2003) 2631-2636.

9) B. Karbakhsh Ravari and M. Nishida: Philos. Mag. 93 (2013) 22792296.

10) B. Karbakhsh Ravari, S. Farjami and M. Nishida: Acta Mater. 69 (2014) 17-29

11) Y. Soejima, S. Motomura, M. Mitsuhara, T. Inamura and M. Nishida: Acta Mater. 103 (2016) 352-360.

12) J. Khalil-Allafi, A. Dlouhy and G. Eggeler: Acta Mater. 50 (2002) 4255-4274.

13) A. Dlouhy, J. Khalil-Allafi and G. Eggeler: Philos. Mag. 83 (2003) 339-363.

14) S. Cao, M. Nishida and D. Schryvers: Acta Mater. 59 (2011) 17801789.

15) S. Miyazaki, Y. Igo and K. Otsuka: Acta Metall. 34 (1986) 2045-2051.

16) T. Tadaki, Y. Nakata and K. Shimizu: Trans. JIM 28 (1987) 883-890.

17) F. Yamashita, M. Wakoh, K. Ishikawa and H. Shibata: Mater. Trans. 58 (2017) 1729-1734 